\title{
Innovative Robot Archetypes for In-Space Construction and Maintenance
}

\author{
Fredrik Rehnmark ${ }^{1}$, Robert O. Ambrose ${ }^{2}$, Brett Kennedy ${ }^{3}$, Myron Diftler $^{2}$, Joshua \\ Mehling $^{2}$, Lyndon Bridgwater ${ }^{2}$, Nicolaus Radford ${ }^{4}$, S. Michael Goza ${ }^{2}$ and \\ Christopher Culbert ${ }^{2}$
}

\author{
${ }^{1}$ Lockheed Martin Space Operations, 2400 NASA Parkway, Houston, TX 77058 \\ ${ }^{2}$ NASA Johnson Space Center, 2101 NASA Parkway, Houston, TX 77058 \\ ${ }^{3}$ Jet Propulsion Laboratory, California Institute of Technology, 4800 Oak Grove Drive, Pasadena, CA 91109 \\ ${ }^{4}$ Oceaneering Space Systems, 16665 Space Center Boulevard, Houston, TX 77058 \\ 281-244-5562; fredrik.rehnmark@lmco.com
}

\begin{abstract}
The space environment presents unique challenges and opportunities in the assembly, inspection and maintenance of orbital and transit spaceflight systems. While conventional Extra-Vehicular Activity (EVA) technology, out of necessity, addresses each of the challenges, relatively few of the opportunities have been exploited due to crew safety and reliability considerations. Extra-Vehicular Robotics (EVR) is one of the least-explored design spaces but offers many exciting innovations transcending the crane-like Space Shuttle and International Space Station Remote Manipulator System (RMS) robots used for berthing, coarse positioning and stabilization. Microgravity environments can support new robotic archetypes with locomotion and manipulation capabilities analogous to undersea creatures. Such diversification could enable the next generation of space science platforms and vehicles that are too large and fragile to launch and deploy as selfcontained payloads. Sinuous manipulators for minimally invasive inspection and repair in confined spaces, soft-stepping climbers with expansive leg reach envelopes and free-flying nanosatellite cameras can access EVA worksites generally not accessible to humans in spacesuits. These and other novel robotic archetypes are presented along with functionality concepts.
\end{abstract}

\section{INTRODUCTION}

Some of the most significant technological challenges facing future space exploration programs arise from practical limitations on what can be launched into orbit around Earth. Space systems must meet mass, volume, durability, safety and many other requirements on the launch pad, effectively limiting their size and complexity. The International Space Station (ISS) provides an important (and expensive) data point illustrating the strengths and limitations of the preintegrated modular assembly approach to building large, complex space structures. The next generation of space vehicles and platforms supporting extended human missions and scientific observations could benefit from more flexible and extensible EVA (Extra-Vehicular Activity) and robotic in-space construction and maintenance capabilities. Examples include telescopes with extremely long focal lengths, expansive orbital power generation facilities, and reconfigurable space vehicles and habitats.

In-space facilities assembled and maintained by human astronauts, such as the Hubble Space Telescope, are extremely expensive to build and operate. Robotically serviceable spacecraft designs offer several advantages, including relaxed safety standards, more economical access and more flexible configurations. Most of today's in-space robots are modified versions of factory manipulators, with minor replacements of materials and control modes that date back to the 60's and 70's. NASA's recently formed Exploration Systems Mission Directorate has expressed interest in developing new archetypes, ranging from novel and highly dexterous manipulation tools to self-assembling systems and robotic free-flyers that may be remotely controlled or automated. Flexible and reusable systems that can be used both for construction and maintenance of general classes of space assets are preferred. Technologies are needed for the entire assembly and maintenance lifecycle, including acquiring/deploying components, achieving alignment, part mating, connection of ancillary equipment, verification of assembly completion, periodic inspection, maintenance, and repair. 
Much experimental work has been done to advance the state-of-the-art in Extra-Vehicular Robotics (EVR) for microgravity environments, yielding robotic free-flyers, dual-arm dexterous servicing systems and novel locomotion schemes. Three novel biologically-inspired robot archetypes for in-space construction and maintenance are presented and discussed herein as "works-in-progress." They include an arachnid climber, a fine manipulation specialist and a minimally invasive tendril. Early prototypes of these systems are already in existence, with specific technology development and performance objectives in mind. The arachnid climber must be able to walk lightly across large space structures and will be refined to impart less than $15 \mathrm{~N}$ climbing forces in 1 -g air-bearing floor tests. The fine manipulation specialist must be flexible enough to handle a range of construction and maintenance tasks with minimal limb reconfiguration. The minimally invasive tendril must be able to access EVA worksites not directly accessible to humans by virtue of its extremely slender 1:1000 diameter to length aspect ratio.

\section{HISTORY}

Recognizing the opportunity to augment human presence in space with cost-effective machines, NASA has investigated a variety of EVR archetypes over the years to assist astronauts conducting EVAs. Building on the success of the Space Shuttle Remote Manipulator System (SRMS) robotic arm, NASA worked with the Canadian Space Agency (CSA) to develop the International Space Station SSRMS. Both devices have performed extraordinarily well in maneuvering large payloads, astronauts, the shuttle, and even occasionally removing a piece of ice.

Early in the Space Station Freedom program, NASA commissioned Martin Marietta to build the Flight Telerobotic Servicer (FTS) (McCain et al., 1991) to provide a mid range robotic capability for handling payloads up to approximately $500 \mathrm{~kg}$, thereby complementing the RMS. The FTS design used specialized end effectors to manipulate robotically serviceable Orbital Replacement Units (ORUs). Extending from its body were two manipulator arms, stereo cameras and a single stabilizing leg. This design and subsequent hardware development represented NASA's first step into serious consideration of robotic archetypes beyond the simple serial chain manipulator system. To meet program requirements, the tasks originally slated for the FTS were eventually reduced and combined with those planned for CSA's Special Purpose Dexterous Manipulator (SPDM). FTS was eventually cancelled and replaced by SPDM, a system currently awaiting launch, but the inspiration for different classes of manipulators continued to motivate NASA designers.

With only a small percentage of Space Station ORUs specifically designed for robotic servicing, NASA has been motivated to explore alternate means to relieve the crew of the considerable EVA maintenance burden associated with them. In the early 90's, Lockheed and NASA engineers at the Johnson Space Center built a testbed to determine if a robot modeled after a human could assist crew with tasks not designed for robots. The Dexterous Anthropomorphic Robotic Testbed (DART) (Li et al., 1996) is a ground-based, teleoperated testbed that helped NASA make this determination. Featuring a pair of 3-fingered hands mounted on 6-jointed robotic arms, DART has sufficient mechanical dexterity to work with a variety of EVA tools, interfaces and materials including tether hooks, wire cutters, hand rails and flexible blankets. The insight gained from DART led to NASA's decision to develop Robonaut (Ambrose et al., 2000), a far more capable space humanoid.

Concurrently, large scale orbital facilities significantly beyond the size of the Space Station have been considered and preliminary designs commissioned. Because of single-launch payload mass and volume constraints and stowage requirements to survive high accelerations, space-based construction is the only option for these power stations, telescopes, and vehicles. Greatly expanded EVA/EVR capabilities will be required to make these constructions feasible. Carnegie Mellon University's Skyworker (Staritz et al., 2001) is a next generation version of the Shuttle and Station space cranes designed to manipulate and transport the large masses that make up these new facilities. Unlike the SSRMS, Skyworker can "walk" along a space structure without requiring specialized grapple points. It is designed to carry a range of masses and maintains a high level of power efficiency by employing a continuous gait that yields a constant velocity for even large payloads. A scaled prototype of Skyworker was successfully demonstrated at Carnegie Mellon.

A radically different class of space robot is the free-flyer micro-satellite that can provide a variety of inspection data and direct camera views to assist astronauts. The Autonomous EVA Robotic Camera (AERCam) prototype "Sprint" was tested during Shuttle mission STS-87 in December, 1997. This prototype performed outstandingly and its derivative Mini-AERCam is now slated as a tool for on-orbit Shuttle Thermal Protection System inspection. The opportunity to augment this useful tool with additional capabilities, including some form of manipulation, is of great interest to the space community. 


\section{MOVING AND WORKING IN MICROGRAVITY ENVIRONMENTS}

Humans living and working in microgravity must learn how to deal with objects that do not stay where they are placed, the absence of weight-induced friction, prolonged momentum effects and the increased manifestation of secondary effects such as Coriolis forces and air currents (intra-vehicular only). The peculiarities of the microgravity environment found on orbit, at libration points and in transit to destinations like the Moon and Mars simplify some tasks, like maneuvering massive, bulky objects but complicate others, like working with both hands while trying to stabilize one's body. However, the forces required to manipulate floating objects in microgravity can be quite small, amounting to only a tiny fraction of the payload's Earthbound weight. Typically, astronauts use handrails, foot restraints, Velcro, tethers, cranes, robotic manipulators and other specialized zero-g devices to aid them in capturing and controlling free-floating objects on orbit (Figure 1).

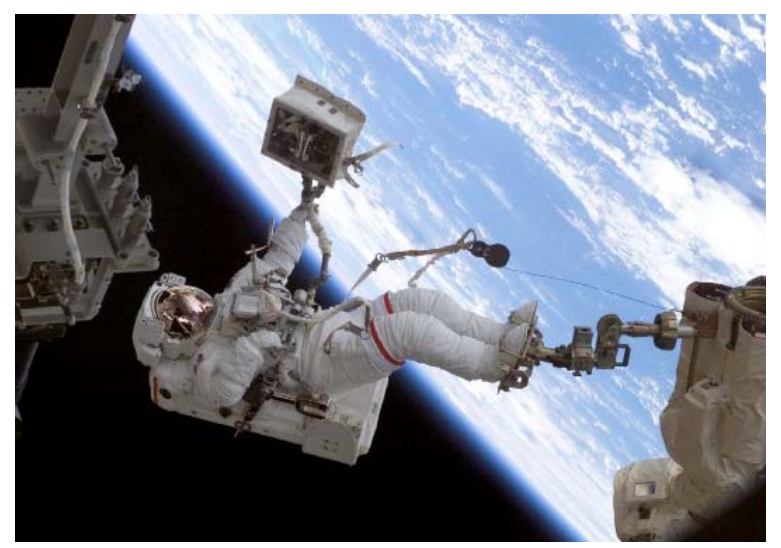

FIGURE 1. Astronaut David Wolf Riding the SSRMS End-Effector in a Foot Restraint on STS 112.

Space walking is poorly named, as it has little in common with how animals walk on Earth. For humans, space walking is more akin to mountain climbing in scuba gear, while parachuting in a freefall: an odd combination of effects and equipment to help people do a demanding job. In order to move about in this environment, a robot must be able to climb autonomously, using gaits that smoothly manage its momentum and that minimize contact forces (walking lightly), while providing for safety in the event of an emergency requiring the system to stop.

Scalability is a key advantage of robotic systems over humans, permitting a high degree of specialization suited to the service environment and task. On the ground, gravity imposes strict restrictions on the scale of a robotic servicer's body compared to its limbs and end-effectors. Such restrictions disappear in zero-g, enabling even greater diversification of robotic morphologies than possible on the ground.

\section{ARACHNID CLIMBER}

Shuttle and ISS EVA translation/stabilization aids are designed to tolerate substantial astronaut-induced loads. EVA handrails along primary translation paths are required to support climbing forces up to $220 \mathrm{lbf}(979 \mathrm{~N})$, corresponding to a one-handed grasp, and combined forces and torques up to $100 \mathrm{lbf}$ and $600 \mathrm{in}-\mathrm{lbf}$ ( $445 \mathrm{~N}$ and $67.8 \mathrm{Nm}$ ), corresponding to a two-handed grasp. EVA crew tether attach points are required to withstand tether forces up to $200 \mathrm{lbf}(890 \mathrm{~N})$ and spacecraft structure within 24" of a primary translation path must survive $200 \mathrm{lbf}(890 \mathrm{~N})$ kick-off loads as well as smaller (but more concentrated) $125 \mathrm{lbf}(556 \mathrm{Nm}$ ) accidental impacts (NASA Johnson Space Center, 1999). Future large scale spacecraft and large aperture arrays may consist of delicate structural frameworks too fragile to survive this kind of abuse.

The bipedal climbing gait of a human astronaut requires small, but non-zero, wrist moments to safely manage momentum in a microgravity environment. In contrast, the multipoint stance of an arachnid's eight legs, with as many as 7 down during a step, allows simply-supported footholds that spread climbing loads more evenly across a space structure and impart no torques. The arachnid anatomy allows a wide range of locomotion options observed in nature (Suter and Wildman, 1999), which include running and galloping across water surfaces. The robotics community has studied general climbing gaits (Bretl, Latombe and Rock, 2003) and eight legged gaits (Roßmann and Pfeiffer, 1998) and has sometimes referred to six legged, hexapod machines as spiders. 
Inspired by the locomotion of spiders, the arachnid archetype specializes in climbing across light and fragile space structures without doing damage. Such a system could carry large payloads, transporting structural materials across an extensive solar array or mirrors across a telescope without significant structural loading. This archetype could also exploit hybrid forms of locomotion such as routing and deploying a "web" of space tethers to cross structural spans where even light forces are unacceptable. A spider-like robot prototype, under development at NASA's Johnson Space Center (JSC), forms the basis for a series of new arachnid class robots. Shown in Figure 2, the prototype was developed to test a pair of legs, develop climbing gaits and experiment with a novel application of passive springs in limb designs. The key metric for measuring the spider technology's advancement will be the minimization of climbing loads.

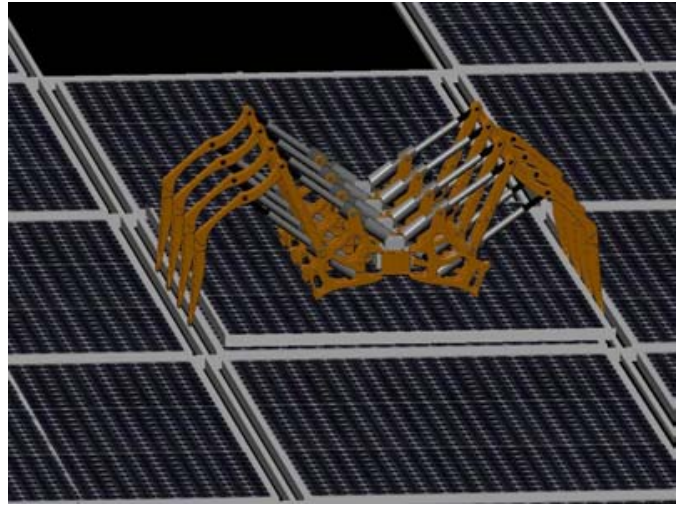

(a) Conceptual Spider Shown Transporting a Panel Element Across a Space Solar Array.

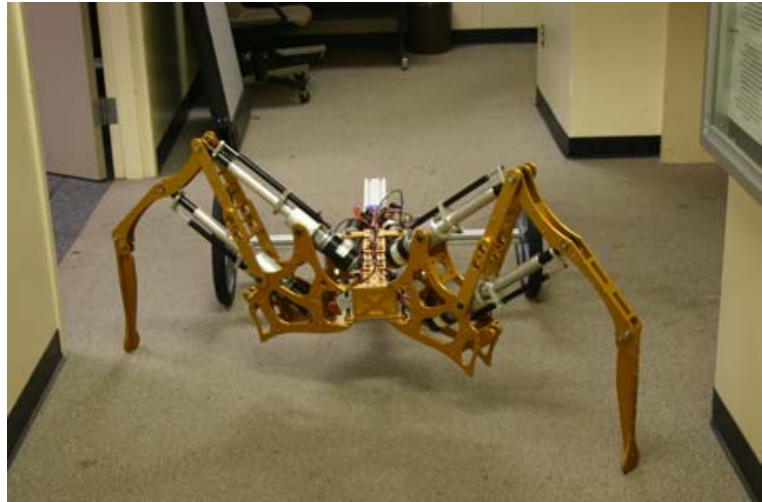

(b) Two-Legged Spider Prototype.

FIGURE 2. Arachnid Climber for Locomotion Across Large, Fragile Space Structures.

The existing system has a number of unique but low TRL (Technology Readiness Level) technologies that will be matured including:

1) High actuator strength to structure strength ratios

2) A high overall strength to weight ratio (25:1 in nominal stance, 10:1 in extreme stance)

3) An elastic energy storage system that further increases strength-to-weight ratios

4) Slender packaging for compact storage during launch

5) A two degree of freedom "thigh" segment that places actuators near the machine's body

6) Hard points on the machine's underbelly for carrying construction materials across spacecraft

7) Arachnid climbing gaits and algorithms matured through simulations and ground tests

A complete, 8-legged arachnid will be developed based on lessons learned during testing of the existing 2-legged prototype. The system will be exercised in a $1 \mathrm{~g}$ climbing and transport experiment for the purpose of measuring contact forces and moments imparted to the climbing surface. The initial goal will be to limit contact forces to $<15 \mathrm{~N}$ (peak) and $<10 \mathrm{~N}$ (average) during planar climbing with the robot's weight offloaded onto an air-bearing floor.

A spider's stride length and mass is well proportioned to the web it weaves. One of the keys to achieving low contact forces and simple gaits for the arachnid climber will be an integrated, modular design of the space structure it will traverse. The geometry, strength and stiffness of the structure will be designed concurrently with the arachnid climber, providing a powerful set of variables for optimization. Meanwhile, structural truss nodes will be designed with specific interfaces for the spider's feet, allowing the robot to translate without touching the more delicate truss rods.

\section{FINE MANIPULATION SPECIALIST}

EVA astronauts working in the vacuum of space face numerous technical challenges in addition to zero-g locomotion. The EMU (External Mobility Unit) space suit glove significantly degrades manual dexterity and tactile feedback while reducing grasp strength and endurance. To be sure, this glove is a technological marvel that protects the astronaut from the harsh space environment. A machine, however, will not need such elaborate protection. Scale matters - a smaller limb with end tooling will be able to reach into tighter confines and handle smaller items like screws and individual wire strands exceeding conventional EVA dexterity. Researchers at the California Institute of Technology's Jet Propulsion 
Laboratory (JPL) have begun to explore an archetype of robot that can climb and handle small objects. Shown in Figure 3, the LEMUR (Limbed Excursion Mechanical Utility Robot) (Kennedy et al., 2001) and LEMUR II have a mix of legs, with interchangeable tools that include cutting, fastening, clinging and walking functions.

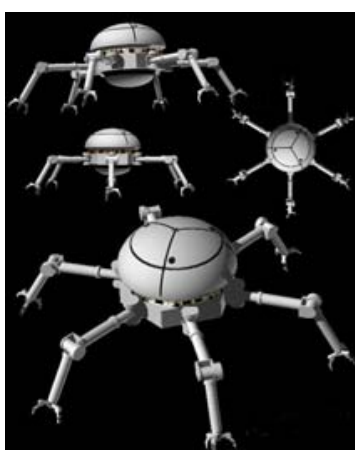

(a) LEMUR Concept Drawing.

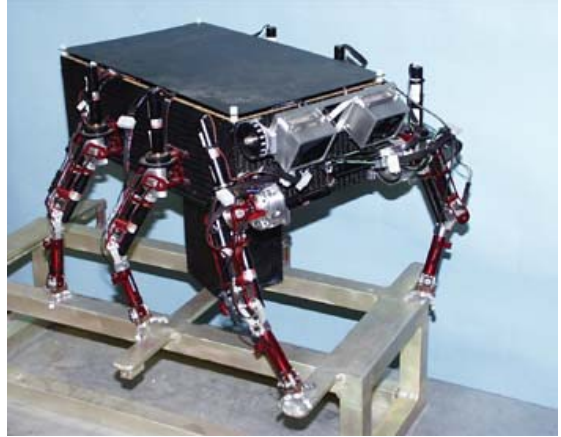

(b) First LEMUR Prototype Climbing.

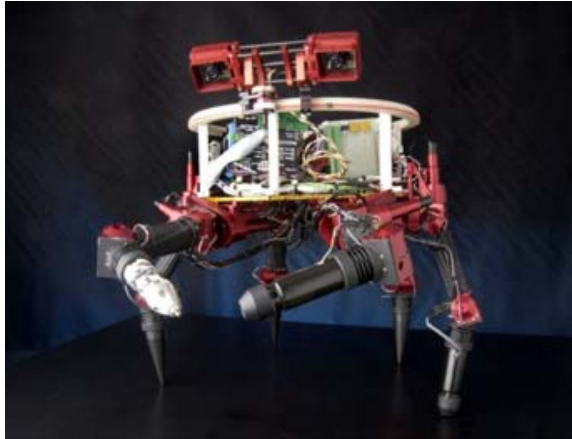

(c) LEMUR II Prototype with Tooling.

FIGURE 3. Three Generations of the LEMUR Design for Fine Manipulation in Space.

Different archetypes are needed to effectively partition the in-space assembly work as well as the resources of mass, power and volume available to EVR systems. In LEMUR's case, the initial assumption is that an archetype is necessary to carry out fine assembly and inspection tasks. Furthermore, this robot should carry a modular set of instruments and tools because its direct, physical interactions with the environment will be more highly specialized across its operational modes (i.e., the "right" tool for the job may vary more for the fine manipulation robot than it would for other archetypes). The nature and complexity of these instruments and tools provide a basis for design variation and future development.

Representing an existence proof, the primate model of multi-mode limbs (particularly mobility and manipulation) with tool-using capability provides a clear design path for the fine manipulation archetype. The robot's limbs should be long relative to body size and limb spacing, resulting in generous, overlapping workspaces. However, the arrangement of the limbs with respect to the body is not so easily determined. Given that top vs. bottom and front vs. back comparisons have less meaning in a microgravity environment, the robot should embody more than bilateral symmetry. Future refinements of the fine manipulation robot would most likely retain the overall axi-symmetric, multi-limbed configuration of LEMUR II to minimize directional dependencies. In fact, through judicious sensor layout, a robot with almost no directional preference could be designed, reducing the need for operationally expensive movements required to reorient the body for locomotion or manipulation tasks. A less obvious advantage of axi-symmetry and long limbs is that any combination of limbs can be brought to bear on a workpiece or other task objective.

The movement mode of the fine manipulation robot will impact its limb count. While the alternating tripod gait of the Earthbound LEMUR is not needed for stability in space, it may be highly advantageous to maintain a three-point contact with space structures. Such a contact pattern distributes reaction forces evenly and allows the choice of force types expressed at individual contacts (i.e., the mix of reaction moments and/or linear forces). Assuming three limbs in contact, one advancing limb and two limbs available for cooperative manipulation, a minimum of six limbs would be required.

\section{MINIMALLY INVASIVE TENDRIL}

A third robot archetype that has great potential for use in the inspection and maintenance of space structures finds its inspiration in the biology of snakes, tentacles, and climbing plants. This new class of long, slender manipulators is optimized for micro-g environments, with a $5 \mathrm{~mm}$ diameter limb that can extend more than $5 \mathrm{~m}$, carrying inspection sensors underneath thermal blankets and deep into complex crevasses, MMOD (MicroMeteoroid Orbital Debris) impact holes and fluid lines found on spacecraft exteriors. Inaccessible to astronauts, these locations could be reached with minimally invasive techniques to verify and inspect structure and embedded systems. Tendril manipulators, similar to the arms of sea creatures like anemones, octopi or squid, will be able to pass into tight confines and through complex geometries. 
Unlike its biological analogues, the tendril can carry sophisticated imaging, diagnostics and repair tools for sealing cracks, soldering wires, probing circuits, etc. but will require novel actuation schemes. The key metric for measuring the tendril technology's advancement will be the manipulator aspect ratio, with an ultimate goal of a $(1: 1000)$ diameter to length ratio. Shown in Figure 4, the proposed tendril will be a coil-deployed, ultra-long limb that could not be considered for operation except in the unique conditions of $0 \mathrm{~g}$. Even the ocean currents at the bottom of the sea would be a challenge for this slender manipulator, but it will thrive in the airless and weightless domain of space. Ground testing of the tendril will require creative use of air-bearing floors, neutral buoyancy pools or testing over limited workspaces while hanging vertically.

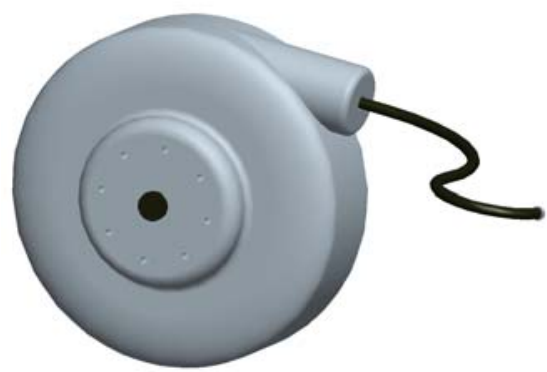

(a). Tendril Coil.

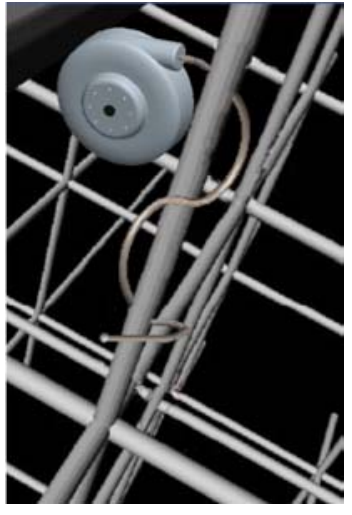

(b). Inspection of Space Structures.

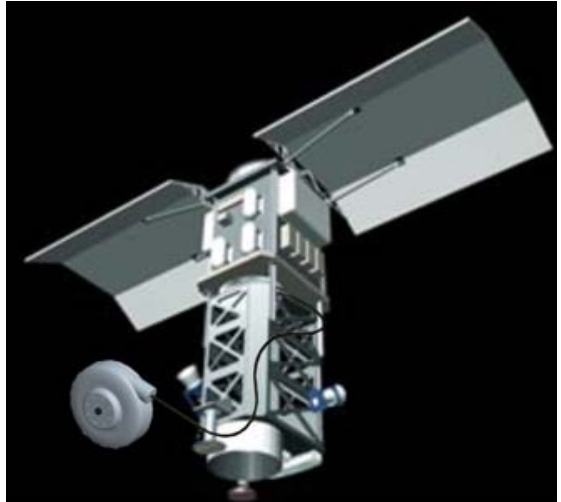

(c). Repair of Satellite Subsystems.

FIGURE 4. Tendril Robot Concepts for Minimally Invasive Inspection and Repair in Space.

Small-scale dexterity and operating within a confined workspace has already been extensively researched with minimally invasive surgery in mind. Commercial devices used in laparoscopic procedures, such as Intuitive Surgical's daVinci system, have dexterous wrists and a range of end effectors that can pass through small ports $(\sim 1 \mathrm{~cm})$ and work inside the body with reduced trauma to patients (www.intuitivesurgical.com). Additionally, actuation of tendril-like, high aspect ratio surgical tools has been investigated with various robotic endoscopes (Maeda et al., 1996), (Ikuta, Tsukamoto and Hirose, 1988), (Slatkin, Burdick and Grundfest, 1995). Here, the ability to control not only the tool tip, but also the bending of the device's length is achieved. Perhaps the most promising device of this class is a recent prototype developed by Simaan, Taylor and Flint (2004) for laryngeal surgery. This device is of interest due to its small diameter $(4.2 \mathrm{~mm})$ and the promise of realizing the high aspect ratios $(1: 100$ and 1:1000) desirable for tendril robots in space applications.

Ultimately, to achieve the desired dexterity for tendril robots used in space construction and maintenance tasks, a simple bending of the device's length is not enough. To effectively perform its tasks, a tendril must be able to make multiple turns and twists around obstacles and through holes. Biology offers numerous examples of this kind of motion control (a snake, the tentacles of an octopus, etc.) but modern technology has yet to produce an actuator that performs as well as natural muscle. Current biologically inspired systems, like the elephant trunk and tentacle robots developed by Walker (2000), do mimic animal behavior fairly well, however. Innovative new actuator designs based on advanced materials such as shape memory alloys, magnetorheological fluids and electroactive polymers are being developed to achieve the extremely slender aspect ratio characteristic of the tendril archetype.

\section{COORDINATED SYSTEMS TEAMING SCENARIO}

Complex in-space construction and maintenance operations require dispersed manipulation, locomotion and sensing capabilities. Some assembly tasks require a combination of force and finesse that can't be delivered by a single assembly agent. Installing a steel girder in a building, for example, is accomplished with the help of a crane to do the heavy lifting while humans at either end of the beam line up the bolt holes and fasten the beam in place. Coordinated, multi-agent teams consisting of human astronauts and heterogeneous robotic systems can meet these needs. Figure 5 illustrates a possible teaming arrangement between the large arachnid climber described earlier and several smaller LEMUR robots capable of fine manipulation. 


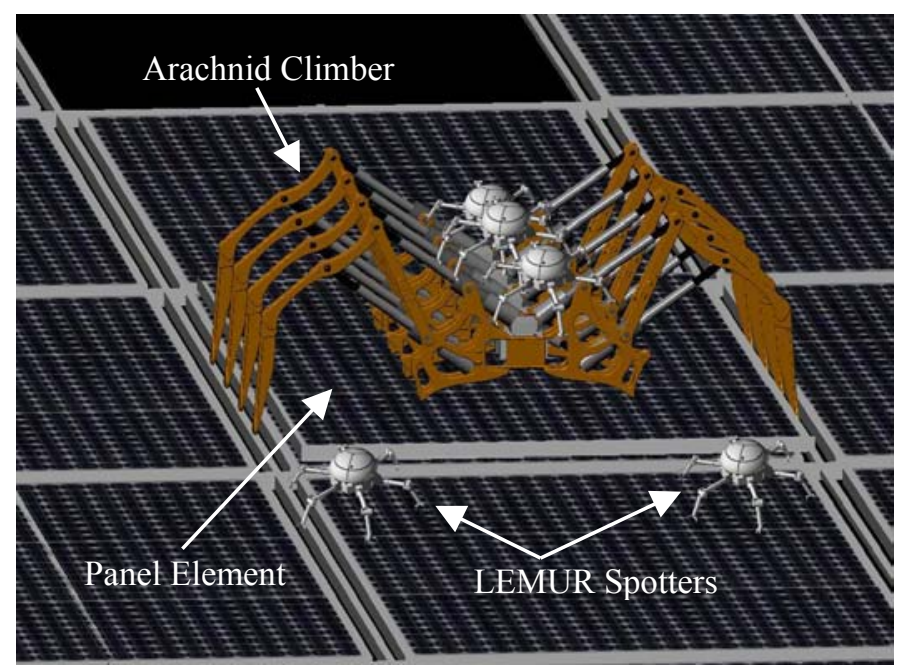

FIGURE 5. Proposed Teaming Arrangement between Large Arachnid Climbing Robot and Numerous LEMUR Spotters.

In this task scenario, the team builds a space solar panel array element-by-element, transporting individual panels across a structural lattice and installing them through a cooperative effort. The spider robot transports each panel element from a cargo carrier (not shown) to its prescribed destination on the array and provides coarse positioning while lowering it into place. Meanwhile, the LEMUR robots act as spotters, providing small but precise correction forces and auxiliary views of critical hardware clearances for alignment and insertion of the panel element. They are also equipped with task-specific tooling used to tighten retaining screws after the panel has been lowered into place. The spider robot, in turn, transports its smaller teammates over the large distances involved in point-to-point translations between worksites on the array.

Computation in the team must necessarily be distributed, because no single agent would be able to coordinate the activities of all the other agents without risking single point failures. This characteristic points to the need for a control architecture and software tools to coordinate complex tasks, especially those that require tight coupling between robots. The architecture must not only sequence tasks across robots, but should also be responsible for determining when there has been a failure and indicate to an operator the steps that must be taken to recover from a contingency. Further, the architecture should allow for a seamless transition between human and automatic control of tasks - the operator should be able to step in and take control of any agent in the system at any time.

Researchers at Carnegie Mellon University have been working in the area of control architectures for task execution/monitoring and "sliding autonomy" specifically targeting NASA's need to assemble structures in space. A current project funded by the Space Solar Power Program is developing a system that enables three heterogeneous robots to autonomously assemble four beams into a structure (Brookshire, Singh and Simmons, 2004). Because such a task requires a large number of small steps, the system can experience a large number of unanticipated contingencies. The control architecture makes it possible for a human operator to assume control of any robot at any time. Additionally, the robots themselves are able to monitor their own success and can signal the operator if they are unable to accomplish any of the tasks assigned to them.

\section{CONCLUSIONS}

The challenges associated with building and operating the large scale space facilities of the future will demand greatly expanded EVA/EVR capabilities. Three novel robot archetypes with applications to in-space construction, maintenance and inspection have been presented along with functionality concepts and a coordinated systems teaming scenario. The arachnid climber, fine manipulation specialist and minimally invasive tendril all meet specific needs in the EVR arena. Although still in the early stages of development, prototypes and proof-of-concept testbeds of these servicing systems already exist and will be exercised in 1-g cooperative assembly demonstrations. In the future, subsystem technologies will be refined in a design-integrate-test spiral open to concurrent developments in related disciplines. This is an effort to meet NASA's evolving needs for new robotic servicing systems to assist in the assembly, inspection and maintenance of space vehicles and platforms being considered for future space exploration programs. 


\section{ACKNOWLEDGMENTS}

This work was made possible by NASA's Exploration Systems Mission Directorate under the Human and Robotic Technologies program.

\section{REFERENCES}

Ambrose, R.O. et al., "Robonaut: NASA's Space Humanoid," IEEE Intelligent Systems and Their Applications 15(4), 57-63 (2000).

Bretl, T., Latombe, C. and Rock, S., "Toward Autonomous Free-Climbing Robots," in proceedings of International Symposium of Robotics Research, Siena, Italy, 2003.

Brookshire, J., Singh, S. and Simmons, R., "Preliminary Results in Sliding Autonomy for Coordinated Teams," in proceedings of 2004 AAAI Spring Symposium, AAAI Press, Palo Alto, California, 2004, pp. 127-132.

Ikuta, K., Tsukamoto, M. and Hirose, S., "Shape Memory Alloy Servo Actuator System with Electric Resistance Feedback and Application for Active Endoscope," in proceedings of IEEE International Conference on Robotics and Automation (ICRA 1988), IEEE Computer Society Press, Philadelphia, Pennsylvania, 1988, pp. 427-430.

Intuitive Surgical, (2003), http://www.intuitivesurgical.com, accessed October 11, 2004.

Kennedy, B. et al. "LEMUR: Legged Excursion Mechanical Utility Rover,” Autonomous Robots 11(3), 201-205 (2001).

Li, L. et al., "Development of a Telepresence Controlled Ambidextrous Robot for Space Applications," in proceedings of IEEE International Conference on Robotics and Automation (ICRA 1996), volume 1, Minneapolis, Minnesota, 1996, pp. 58-63.

Maeda, S. et al., "Active Endoscope with SMA (Shape Memory Alloy) Coil Springs," in proceedings of IEEE International Workshop on Micro Electro Mechanical Systems (MEMS 1996), San Diego, California, 1996, pp. 290-295.

McCain, H.G. et al., "Flight Telerobotic Servicer: the Design and Evolution of a Dexterous Space Telerobot," in proceedings of Telesystems Conference 1991, Atlanta, Georgia, 1991, pp. 385-390.

NASA Johnson Space Center, Extravehicular Activity (EVA) Standard Interface Control Document, International Space Station Program Document SSP 30256:001 Revision G, Houston, Texas, 1999.

Roßmann, T. and Pfeiffer, F., "Control of an Eight-Legged Pipe Crawling Robot," in Lecture Notes in Control and Information Sciences 232, edited by Alicia Casals and Anibal T. de Almeida, Springer, 1998, pp. 335-346.

Simaan, N., Taylor, R. and Flint, P., "A Dexterous System for Laryngeal Surgery - Multi-Backbone Bending Snake-Like Slaves for Teleoperated Dexterous Surgical Tool Manipulation," in proceedings of IEEE International Conference on Robotics and Automation (ICRA 2004), New Orleans, Louisiana, 2004, pp. 351-357.

Slatkin, A.B., Burdick, J. and Grundfest, W., "The Development of a Robotic Endoscope," in proceedings of IEEE International Conference on Intelligent Robots and Systems, volume 2, IEEE Computer Society Press, Pittsburgh, Pennsylvania, 1995, pp. 162-171.

Staritz, P.J. et al., "Skyworker: a Robot for Assembly, Inspection and Maintenance of Large Scale Orbital Facilities," in proceedings of IEEE International Conference on Robotics and Automation (ICRA 2001), Seoul, Korea, 2001, pp. 4180-4185.

Suter, R.B. and Wildman, H., "Locomotion on the Water Surface: Hydrodynamic Constraints on Rowing Velocity Require a Gait Change," Journal of Experimental Biology 202, 2771-2785 (1999).

Walker, I.D., "Some Issues in Creating 'Invertebrate' Robots," in proceedings of International Symposium on Adaptive Motion of Animals and Machines, Montreal, Canada, 2000. 